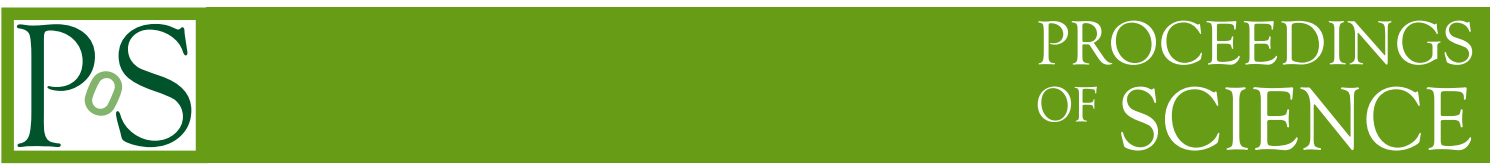

\title{
Results of the sixth Waseda Nasu radio transient survey using a new system
}

\author{
Sumiko Kida \\ Waseda University \\ Department of Science, School of Education, Waseda University, 1-6-1 Nishi-Waseda, \\ Shinjuku-ku, Tokyo 169-8050, Japan \\ E-mail: sumiko_kesuou.waseda.jp
}

\author{
Kuniyuki Asuma ${ }^{a}$, Takahiro Aoki $^{a}$, Ryota Hiruma $^{b}$ and Tsuneaki Daishido ${ }^{b}$ \\ ${ }^{a}$ Waseda University \\ Department of Pure and Applied Physics, Graduate School of Science and Engineering, Waseda \\ University, 3-4-1 Okubo, Shinjuku-ku, Tokyo 169-8555, Japan \\ ${ }^{b}$ Waseda University \\ Department of Science, School of Education, Waseda University, 1-6-1 Nishi-Waseda, \\ Shinjuku-ku, Tokyo 169-8050, Japan
}

\begin{abstract}
Radio transient surveys have been carried out at $1.4 \mathrm{GHz}$ by using eight 20 -m dishes at the Waseda Nasu Observatory since 2004. A new observation system was introduced in January 2010. In the new system, a space-based Fast Fourier Transform processor that consisted of a two-step Radix4 based on a Field-Programmable Gate Array (FPGA) was introduced; by using this processor, complex A/D conversions with Nyquist rate sampling $(20 \mathrm{MHz})$ could be carried out. The Nyquist rate sampling facilitated the effective integration of sampling data. A regular wide-field survey using the new system was started in April 2010. This survey is the sixth survey that has been carried out since 2004. A new FFT processor enabled the detection of a hitherto undetected fringe of weak flux, with high $\mathrm{S} / \mathrm{N}$.
\end{abstract}

25th Texas Symposium on Relativistic Astrophysics

December 6-10, 2010

Heidelberg, Germany 


\section{A space-based fast Fourier transform processor}

The Waseda Nasu observatory (WNO) was constructed by Waseda University (Fig. 1). The WNO consists of eight 20-m diameter spherical dish antennas that are arrayed from east to west, and can observe continuously for $24 \mathrm{~h}$ as two elements $\times$ four interferometers. Four declinations can be observed simultaneously. Our observation method is drift scanning. The base length of each two-element interferometer is $84 \mathrm{~m}$. The half-power beam width (HPBW) is approximately $0.6^{\circ}$. We prioritized wide-field observation over sensitivity. The observation frequency was 1.42 $\mathrm{GHz}$ and the bandwidth was $20 \mathrm{MHz}$. The observable declination is $32^{\circ}<\delta<42^{\circ}$. The system noise temperature per element is approximately $80 \mathrm{~K}$. Additionally, one $30-\mathrm{m}$ diameter spherical dish antennas was constructed. A 30-m antenna can be driven in the direction of the elevation and the direction of azimuthal. A 30-m antenna can observe the same coordinates many times in a day though eight $20-\mathrm{m}$ antennas can observe the same coordinates only once a day. The observation frequency and the bandwidth are the same as eight $20-\mathrm{m}$ antennas. The observable declination is $19^{\circ}<\delta<55^{\circ}$.

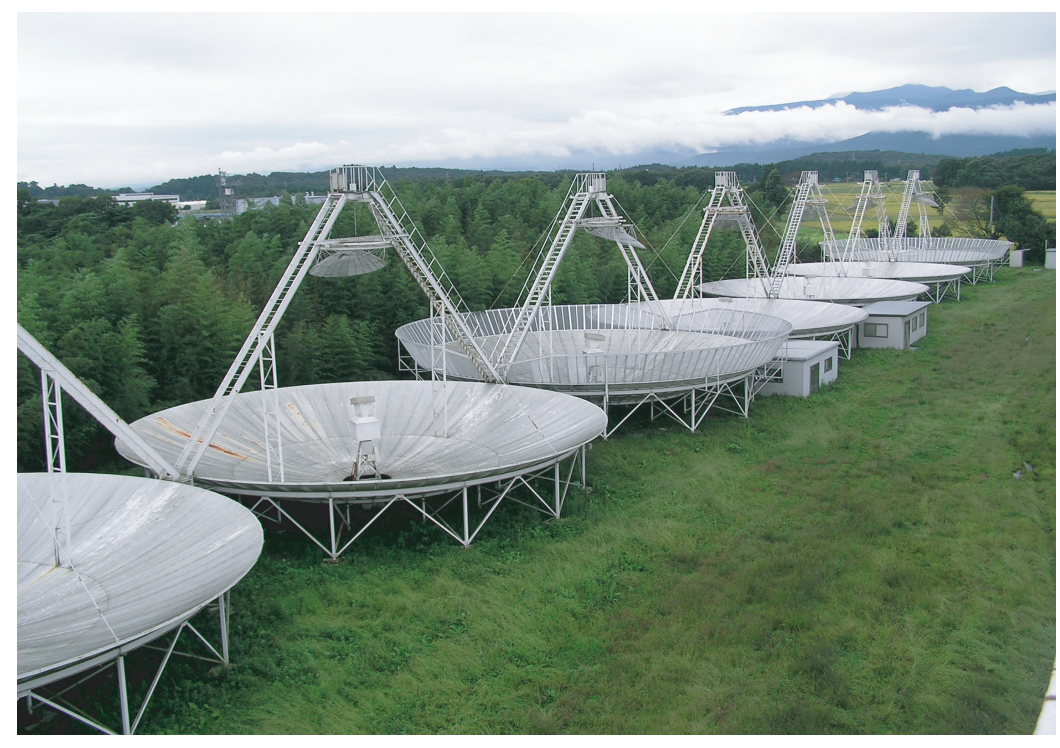

Figure 1: A panorama of the WNO.

Since 2004, radio surveys have been carried out at $1.4 \mathrm{GHz}$ using the eight 20 -m dishes at the WNO. In the old system, the signal received by two antennas was added in an analog manner. The added signal then passed through a diode detector and was digitized with an analog-to-digital (A/D) converter at a sampling rate of $100 \mathrm{~Hz}$. The signal was then recorded on a hard disk after the integration of 100 points. Observations were made with a $1 \mathrm{~Hz}$ phase switch that generates two different data (off-switch and on-switch). The fringe data were produced by subtracting the off-switch data from the on-switch data[1].

A new observation system was introduced in January 2010. In the new system, a space-based fast Fourier transform (FFT) processor that consisted of a two-step Radix-4 based on a FieldProgrammable Gate Array (FPGA) was introduced; with the use of this processor, complex A/D 
conversions with a Nyquist rate sampling $(20 \mathrm{MHz})$ were possible. The Nyquist rate sampling facilitated effective integration of sampling data. Transients of the order of hundreds of millijanskys can be detected using the new system; these transients were not detected by the old system because they were buried in the noise generated in the old system. The FFT processor has three structures. In the front, the delay for the coherence maintenance of the signal is supplemented. The delay is built into the rotator of the first step of the FFT processor. Swinging of the DC offset is removed according to the difference. In the middle, the FFT processing is done with the second two-step Radix-4. In the back, the complex output is converted into the power. The power output is integrated and recorded.

The observation mode changes by replacing the coefficient of Radix-4.

Mode 1 Four independent two-element interferometers

Mode 2 Two independent four-element interferometers

Mode 3 Eight-element interferometers

In this paper, I report a mode 1 observation result.

\section{The sixth survey}

The sixth survey was carried out in April-September 2010. We observed the radio sources every $0.5^{\circ}$ based on a datum line of declination $32.0^{\circ}$. The positional error of declination was approximately $0.6^{\circ}$ because it depended on the half-power beam width (HPBW). The positional error of right ascension was approximately $\pm 20^{\prime \prime}$. Figure 2 shows contour map of the sixth survey.

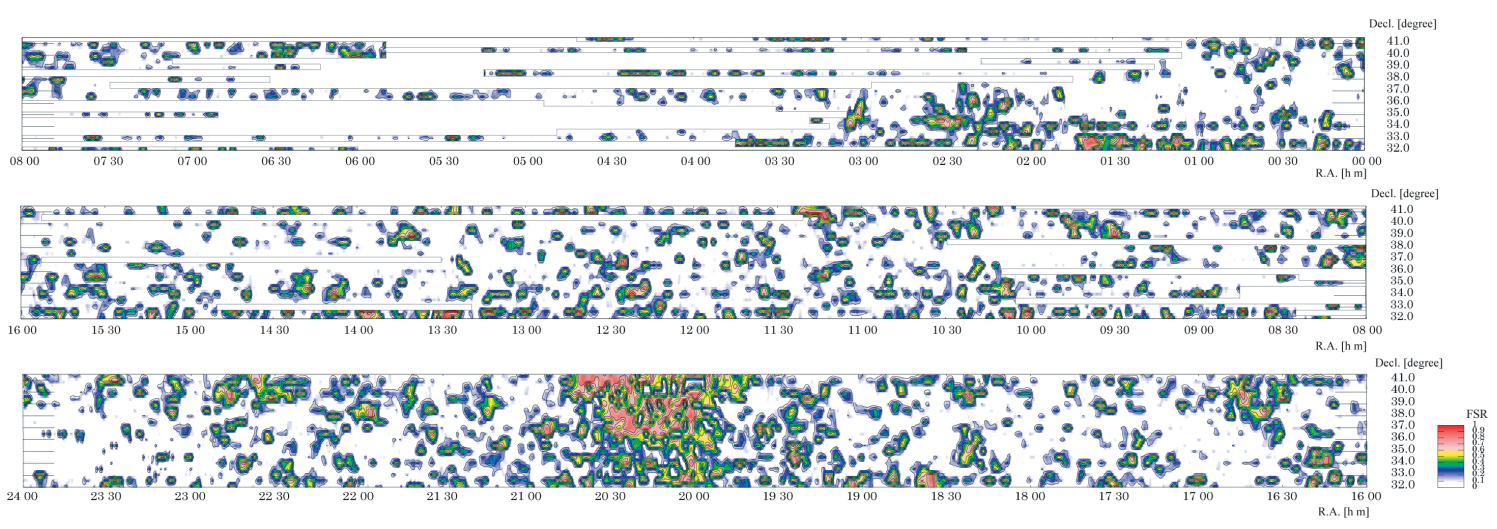

Figure 2: The contour map of the sixth survey. $F S R \geq 0.14$ is displayed as a contour. The white square is the data lack by the influence of sun. The declination is intervals of 0.5 degrees. The contour map was produced based on the following definitions: $F S R=\frac{F(4)}{\sum_{i=1}^{N}}$. Here, $F N R$ is the fringe-to-noise ratio, $N$ is the number of data items, $F(i)$ is the power spectrum, and $F(4)$ is the power spectrum of the fringe frequency. The value of $N$ is four times the fringe cycle. The fringe cycle was $40-45$ seconds at declinations of $32^{\circ}-42^{\circ}$. Therefore, $\mathrm{N}$ was set to $160-180$. 
In an old system, the detection limit based on the integration for ten days was approximately $400 \mathrm{mJy}$ on the average. In an new system, the detection limit based on the integration for ten days is approximately $150 \mathrm{mJy}$. The number of steady radio sources that were able to be detected compared with the first map[2] has increased by four-five times. Though the detection of the weak variable became possible, the confusion must consider more than before carefully.

A new FFT processor enabled the detection of a hitherto undetected fringe of weak flux, with high S/N. Improved observation technology in the other observation mode and continuous observation with the new FFT processor is expected in the future. In addition, cooperation with a 30-m antenna is planned. A variety of observation methods are planned with eight $20-\mathrm{m}$ antennas and one 30-m antenna.

\section{References}

[1] Kuniyoshi, M., et al. 2007, PASP, 119, 122

[2] Takefuji, K., et al. 2008, PASP, 120, 339 